Mots. Les langages du politique

\title{
Esthétique de la limite et dialectique de l'émotion
}

Esthetics of limits and dialectics of emotions

Estética del límite y dialéctica de la emoción

\section{Bernard Lamizet}

\section{OpenEdition}

Journals

Édition électronique

URL : https://journals.openedition.org/mots/3103

DOI : $10.4000 /$ mots.3103

ISSN : 1960-6001

\section{Éditeur}

ENS Éditions

\section{Édition imprimée}

Date de publication : 1 juillet 2004

Pagination : $35-45$

ISBN : 2-84788-057-7

ISSN : 0243-6450

\section{Référence électronique}

Bernard Lamizet, «Esthétique de la limite et dialectique de l'émotion ». Mots. Les langages du politique [En ligne], 75 | 2004, mis en ligne le 22 avril 2008, consulté le 23 avril 2022. URL : http:// journals.openedition.org/mots/3103; DOI : https://doi.org/10.4000/mots.3103 


\section{Esthétique de la limite et dialectique de l'émotion}

La question de la représentation de l'émotion dans les médias est le lieu d'une contradiction majeure, et, comme toute contradiction, sans doute est-elle de nature à nous aider à comprendre les structures et les impératifs de la communication. En effet, le discours des médias est, par définition, un discours politique de nature à organiser et à représenter des logiques d'appartenance et de sociabilité. À l'inverse, une émotion appartient au propre de la subjectivité. C'est le sujet singulier qui l'éprouve ou qui en suggère une à autrui; la connaissance des émotions ne relève pas de notre expérience politique de la sociabilité, mais bien de notre expérience de la subjectivité et de notre savoir sur la dimension singulière de la personnalité. Dans l'information, dans les images et les écrits dont est faite notre culture, la représentation de l'émotion s'inscrit dans une esthétique du sujet dans sa singularité.

\section{L'émotion, figure de la singularité du sujet}

L'émotion est le siège du «réel du sujet», c'est-à-dire de ce en quoi il ne saurait s'identifier symboliquement à l'autre. Elle échappe à toute spécularité. On peut même désigner par ce concept l'ensemble des pulsions et des dynamiques qui structurent le réel de l'expérience de la subjectivité, l'expérience d'un sujet ne pouvant se confondre avec celle d'un autre. L'identité ne s'y vit pas comme une médiation, c'est-à-dire comme une dialectique entre la dimension singulière du sujet et sa dimension collective d'appartenance et de sociabilité: elle ne peut se penser que comme une expérience de la singularité.

Les informations consacrées aux évènements du 11 septembre ${ }^{2}$ mettent en œuvre toute une rhétorique qui se fonde sur des portraits ou sur des documents

1. Institut d'études politiques - Université Lumière, Lyon 2 - bernard.lamizet@univ-lyon2.fr

2. On désigne ainsi la destruction, le 11 septembre 2001, des tours du World Trade Center, à New York, percutées par des avions-suicides, ainsi que l'ensemble des évènements liés à cet attentat. 
concernant des sujets singuliers, nommés : ainsi, «Afiz, l'orphelin de la guerre sainte $»^{3}$ ou encore «Aziza, une esclave afghane de sept ans $»^{4}$. Autre exemple, dans un autre contexte: lisons l'article de Gilles Paris ${ }^{5}$ consacré à un petit palestinien tué par des balles de soldats israéliens. Ce qui produit l'émotion dans ce texte, c'est l'extrême distance entre la dimension politique et institutionnelle de la situation (l'occupation de la Palestine par Israël et les actes de l'armée d'occupation) et l'absence de dimension politique de la vie et de la mort de l'enfant: "L'oncle de Mohamad, Ziad, se souvient d'un enfant aimant la plage et les animaux et particulièrement les quatre oiseaux de la maison offerts par un instituteur de son école». L'émotion est issue, ici, du fait que l'on est sensible au «réel» du sujet: elle vient de la distance maximale établie entre le personnage lui-même, l'enfant, et l'appartenance sociale qu'il représente symboliquement et institutionnellement (c'est un Palestinien). La minutie de la description des lieux nous figure l'espace, elle en fait un espace que nous pouvons imaginer et qui peut servir de cadre narratif à un récit d'identification.

Pour accéder à la maison, lit-on dans l'article, il faut gravir les trois marches de béton

d'un escalier qui part sur la droite. La porte de fer est peinte en blanc comme les murs,

[...]. (Le Monde, 14 octobre 2001)

Rien d'exotique, d'éloigné, dans ce paysage. Il s'agit d'un espace dans lequel nous allons, a priori, pouvoir nous identifier facilement aux personnages de ce qui deviendra une tragédie. Celle-ci sera d'autant plus sensible qu'elle se sera déroulée dans un espace qui pourrait être notre maison, que nous pouvons reconnaitre, presque percevoir, grâce à ce luxe de description, jusqu'à ce détail poignant dans le romantisme populaire qu'il dispense ${ }^{6}:$ «À un mur est accrochée une cage. Trois oiseaux chantonnent».

Quand ils expriment ou représentent la médiation, les médias cessent d'exercer leur fonction de dialectique entre le singulier et le collectif - entre la subjectivité et l'appartenance. Cette représentation entend susciter, de la part du lecteur, une adhésion immédiate, sans distance critique. Dans Le Monde du 11 octobre 2000 , le reportage consacré à la mort du petit palestinien n'est pas politique: il crée l'émotion au cœur du politique. On pourrait parler, dans ce cas, d'une singularisation de l'information, d'une forme de «démédiation». Lors de la représentation de l'émotion, apparait une contradiction, à tout le

3. Le Monde, $14-15$ oct. 2001.

4. Le Monde, 24-oct. 2001.

5. Le Monde, 11-oct. 2000.

6. Peut-être même peut-on trouver, dans ce lien entre la simplicité de la description des lieux et la puissance d'émotion du discours, une interprétation de la règle de l'unité de lieu dans la tragédie classique. 
moins une tension forte, entre les logiques singulières de la subjectivité et les logiques collectives et institutionnelles de la sociabilité. Le Monde du 31 janvier 2001 fait ainsi le récit de la passion douloureuse d'une mère lors du procès en assises de ceux qui sont accusés du meurtre de son fils. "Quand le service d'ordre est sommé de l'évacuer, elle se lève et quitte la salle d'elle-même pour aller crier sa douleur au bout d'un long couloir ${ }^{7}$.» Le procès instaure une tension, voire un conflit (qui ira jusqu'à l'évacuation) entre la singularité du personnage et la dimension collective, indistincte, du dispositif institutionnel auquel il est confronté.

\section{L'émotion, suspension de la dimension sociale de la spécularité}

De telles représentations situent l'émotion dans une stratégie de suspension temporaire des appartenances sociales. Ce processus est comparable à la suspension cartésienne du jugement ou à «l'épochè» husserlienne. La représentation de l'émotion suspend notre sociabilité. Le média n'est plus voué à signifier une appartenance ou un mode de sociabilité, mais à faire apparaitre ce qui échappe à l'indistinction, ce qui échappe à une dimension collective.

Le récit, dans Le Monde du 27 janvier 2001, d'une catastrophe survenue en Inde fait apparaitre la contradiction entre les deux dimensions du fait divers: à la fois marquant par son ampleur, soulignée par le titre ("L'Inde frappée par l'un de ses séismes les plus meurtriers depuis un siècle»), et émouvant par sa singularité, comme le montre le début de l'article, centré sur une image ponctuelle:

Sous le feu de puissants projecteurs, l'homme, le regard anxieux, fixe l'énorme dalle qu'une grue tente pour la troisième fois de soulever. Sa femme, Parveen, 40 ans, n'a pas eu le temps de quitter leur appartement et git sous une montagne de gravats. Trois corps ont déjà été retirés de cet immeuble moderne de cinq étages qui, comme ses voisins encore debout, reposait sur des pilotis de ciment.

La dimension singulière de l'évènement est marquée par la désignation de la femme, son prénom, son âge, qui font d'elle une «personne». L'émotion passe par la suspension des structures politiques, institutionnelles, sociales, qui, dans une situation comme celle-là, ne signifient plus rien pour les acteurs représentés.

7. On remarquera, dans cet énoncé, une rhétorique très significative reposant sur un transfert d'image: le couloir est présenté comme long, ce qui apporte peu au texte mais fait porter sur la description des lieux une figuration de l'intensité d'émotion représentée dans le personnage. 
Au lieu de renvoyer à leurs lecteurs l'image d'une appartenance collective, les médias révèlent l'image de personnes singulières, prises dans des situations qu'elles ne maitrisent pas et qui, de ce fait, accentuent leur isolement. C'est ainsi que, dans le récit de la catastrophe en Inde, on peut lire des propos qui représentent l'institution dans la situation tragique ainsi survenue:

Le premier ministre, Atal Bihari Vajpayee, a réuni un cabinet d'urgence vendredi soir, et a appelé ses concitoyens à se rassembler pour combattre cette calamité qui nous atteint. (Le Monde, 27 janvier 2001)

À travers ces propos, le personnage politique du Premier ministre n'est pas présenté dans les formes et dans la pratique d'une institution. Ses paroles sont moins fondées sur l'engagement politique que sur l'émotion éprouvée («cette calamité qui nous atteint»). Sans doute faut-il interpréter le nous du premier ministre comme la reconnaissance de son appartenance à une communauté de personnes, moins caractérisées par leur citoyenneté que par leur confrontation à un même malheur.

\section{L'émotion dans l'expérience esthétique}

En éprouvant une émotion, nous assumons notre singularité en nous appropriant pleinement l'expérience $"$ esthétique » ${ }^{8}$. Si l'art repose sur l'expérience des perceptions et d'un travail des sens, c'est pour instituer, au cœur de la culture, une suspension de la dimension collective des sujets de la sociabilité.

\section{L'image: l'esthétisation de l'évènement}

À la suite d'un évènement sanglant dans les territoires palestiniens occupés par Israël, Libération publie, en première page ${ }^{9}$, la photo d'un militant palestinien les mains couvertes de sang, après le lynchage de trois soldats israéliens. On peut analyser cette première page en trois éléments qui concourent, chacun de façon spécifique, à la mise en scène de l'émotion.

Le premier élément est, bien sûr, l'élément central : la fenêtre, à laquelle apparait un jeune en train de crier, montrant ses mains ouvertes tachées de sang. Toute la page est ordonnée autour de cette fenêtre, car c'est au visage de l'homme

8. Esthétique est issu du grec aisthanesthai, «percevoir». Ce verbe est ce qu'on appelle, en morphologie grecque, un moyen, c'est-à-dire une forme verbale, sensiblement équivalente à notre verbe réfléchi, qui accentue encore la dimension singulière de ce qu'il exprime.

9. Libération, 13 octobre 2000. 
que le lecteur se trouve confronté comme à l'image d'un miroir, ce personnage lui renvoyant, de façon immédiate, l'horreur de la scène, à la fois par le sang et par la folie qui se peint dans son regard. Cette fenêtre vient marquer le fond du décor de l'illustration - comme s'il s'agissait d'une fenêtre ouverte à nos propres regards - l'émotion venant, pour commencer, de ce que l'on peut appeler la médiation du décor, celui-ci faisant l'objet d'une reconnaissance et d'une appropriation par le lecteur.

Le second élément de la photo est le groupe de manifestants qui font face au personnage, mains et bras levés, vraisemblablement en criant des slogans. Ils se trouvent, en fait, dans la même position que le lecteur, puisque nous les voyons de dos. Ils occupent le bas de l'image (donc la partie de la scène censée être la plus proche de nous); le lecteur pourrait s'identifier à eux, car il est, comme eux, en situation de spectateur. En fait, l'émotion ici représentée comme éprouvée par la foule du premier plan nait d'un face-à-face entre elle et l'homme à la fenêtre qui semble les unir dans un même mouvement, les fondant, sous nos yeux, en un spectacle qui envahit l'espace public. Quelle émotion nous est donc transmise? Ni celle de l'homme, ni celle de la foule. Cette mise en scène instaure une distance, un clivage, entre deux émotions: celle, donnée à voir, qui est éprouvée en même temps par la foule et l'homme à la fenêtre, et celle que nous pouvons éprouver comme lecteurs de l'image et du texte. La scène photographiée a aussi un décor en arrière-fond: il s'agit du mur de ce que la légende nous indique comme un commissariat de police. Ce renseignement donne sens : envahi par ses assaillants, il vient d'être le lieu du meurtre de ses occupants. Tout autour, on hurle à la mort. Le journal commente la scène par son titre : «Vent de haine».

\section{Les titres: l'esthétisation de l'écrit}

Le titre, on le sait, a une fonction éminente dans la construction de l'opinion et dans l'information sur l'évènement. Il a la fonction d'un écrit faisant image. À la différence des textes, il se perçoit d'un bloc, immédiatement, d'un seul regard du lecteur qui parcourt son journal de page en page et, donc, de titre en titre. Par ailleurs, il représente l'information de base, en lui donnant la consistance visuelle d'une organisation iconique de l'espace rédactionnel. Dans le journal, les titres sont là moins pour expliciter que pour construire un espace symbolique en le jalonnant de points de repère. Dans son immédiateté, il suspend la linéarité du discours. Le groupe nominal Vent de haine, sans verbe ni adverbe donc sans temporalité explicite, s'extrait, comme l'image qui l'accompagne, de la linéarité de la découverte progressive de l'information, pour produire, à l'intention du lecteur, l'élément linguistique d'une discursivité immé- 
diate. Dans Le Monde ${ }^{10}$, on peut lire un autre titre: «Muhamad, simple enfant de Gaza». La seule mention du nom de l'enfant tué, Muhamad, et de son expansion, simple enfant de Gaza, représente davantage une évocation, presque une incantation, une plainte, qu'une information sur le drame lui-même.

\section{Le portrait: l'esthétisation des acteurs}

Le portrait, d'abord, rend le personnage identifiable; en le montrant de face, il nous le fait concevoir comme un acteur singulier de l'évènement, reconnaissable à ses traits, à son regard, que nous pouvons voir, à son attitude, que nous pouvons interpréter. Ensuite, le portrait fait apparaitre sur les traits du personnage une émotion qui lui sera propre, en rendant son regard interprétable. Dans ces conditions, l'émotion peinte sur le visage du personnage peut faire l'objet d'une transmission au lecteur de l'image. En rendant reconnaissable l'émotion qui se lit sur les traits du personnage, le portrait donne à son visage la consistance d'un signe. C'est pourquoi le portrait sert à illustrer les informations relatives à l'évènement représenté. Enfin, le portrait sert de centre symbolique à l'image: il condense sur lui l'attention du lecteur, précisément en raison de sa dimension de médiation. En imposant au lecteur de rechercher le regard du personnage qu'il représente, en vue de son identification, il détermine un sens de lecture de l'ensemble de l'image dans laquelle il s'inscrit, construisant, avec le lecteur, grâce la mise en scène esthétique de l'image, une intersubjectivité de nature à impliquer ce dernier.

\section{La rhétorique de l'émotion}

Pour mieux comprendre les logiques rhétoriques de l'émotion, évoquons quelques lignes d'un compte rendu du procès du «tueur en série» Guy Georges. Il s'agit de la plaidoirie de l'avocate de l'inculpé, après les aveux publics formulés par celui-ci en pleine audience:

Il a avoué parce qu'il savait que sa position n'était plus tenable, il avait quelque chose à dire. Notre position était difficile, douloureuse, mais il fallait y arriver, car nous sommes aussi des passeurs. À ce moment-là, il est revenu dans le monde des humains, et ça n'était pas du théâtre. Le fait de passer d'une défense dure à un rôle d'accompagnateurs ne s'est pas fait pour nous sans interrogations, ça ne se fait pas dans le jeu, mais dans l'humanité. (Le Monde, 6 avril 2001)

Dans ce moment particulièrement fort de la plaidoirie de l'avocate, au cours d'une audience très tendue, la rhétorique fait apparaitre dans toute sa dureté le

10. Le Monde du 11 octobre 2000, p. 16. 
réel des acteurs en présence. Dans ce réel, nous sommes seuls, et c'est ce que révèle cette rhétorique de la tension. L'avocate clame, elle fait état d'une situation douloureuse. Quelque temps auparavant, lors d'une autre audience, elle s'était mise à pleurer :

Me Frédérique Pons craque. Sur le banc de la défense, au procès de Guy Georges, le présumé tueur en série de l'Est parisien, l'un de ses avocats pleure. Il est 16h30, le vendredi 23 mars, devant la cour d'assises de Paris. Depuis le début de l'après-midi, la tension est à son comble dans le prétoire. (Le Monde, 26 mars 2001)

Il faut ici la suspension du rapport à l'autre pour que la parole soit rendue à la matérialité, en quelque sorte brute, de sa consistance formelle: dans la situation rhétorique particulière où nous sommes, il faut que l'autre ne soit pas là pour qu'il y ait, justement, émotion, parce que l'énonciateur se retrouve seul. C'est le sens de la phrase "Me Frédérique Pons craque». Il s'agit d'une véritable fracture de la continuité énonciative de la communication. En situation rhétorique, les mots ne sont pas renvoyés à ce qu'ils représentent dans l'intersubjectivité, ils le sont à la consistance réelle de leur effet et de leur instrumentalité dans la stratégie d'un acteur impliqué dans la situation.

\section{L'émotion suspend les médiations dans le discours politique}

Faire appel à l'émotion du public revient à substituer des réactions et des conduites immédiates à la rigueur et à la consistance d'un discours qui ferait de son énonciateur un acteur conscient et engagé. On peut citer en particulier, sur ce plan, la rhétorique de toute propagande - comme, par exemple, les discours populistes, qui reposent sur la mise en évidence de la dimension singulière de la culpabilité ${ }^{11}$ et de l'action. Dans les rhétoriques populistes, la figure de l'émotion prend la forme du transfert, singulier, donc plus ou moins affectif, sur la personne du chef, qui suscite l'émotion de ses partisans. On peut citer l'exemple de la vénération de Staline et de la soumission au totalitarisme, dénoncées par Nikita Mikhalkov dans Soleil trompeur (1994).

Figurée sur la scène politique, l'émotion est aussi un déchainement de passions irréductibles au fait institutionnel:

11. Un bon exemple de cette substitution de la figure de la culpabilité à celle de la responsabilité est donné par la rhétorique de Philippe Pétain, qui se pose, par la figure du père, en initiateur d'une nouvelle morale destinée à redresser les torts causés à la France par les Français eux-mêmes (voir G. Miller, 1975, Les pousse-au-jouir du maréchal Pétain, Paris, Seuil, p. 32-51: «L'exode, dit Pétain, est un châtiment» p. 18). 
Multidimensionnel, écrit un éditorial du Monde ${ }^{12}$, le conflit israélo-palestinien est plus incontrôlable que jamais lorsqu'il dérape sur sa composante religieuse. Il libère alors des passions que le discours politique le plus sage ne peut apaiser.

Dans le discours politique, la mise en scène de l'émotion a plusieurs significations. Tout d'abord, elle individualise l'énonciation. Elle signifie que l'énonciateur assume une légitimité à exprimer sa singularité, sous la forme de l'émotion qu'il éprouve, et confère une légitimité toute personnelle à ce qu'il donne pour l'expression de sa propre subjectivité dans l'espace public. Daniel Cohn-Bendit affirme, par exemple ${ }^{13}:$ «... l'Europe, c'est pour moi une vision, un rêve, je pourrais même dire une des dernières utopies pour laquelle il vaut la peine de se battre ». Ces quelques mots font reprendre conscience aux acteurs politiques de la singularité individuelle de leur engagement: c'est le sens des mots affectifs - une vision, un rêve, une utopie - qui définissent l'engagement européen de l'énonciateur.

L'émotion en politique, visant à ne plus mettre en scène l'énonciateur mais son destinataire, fait de la communication politique une communication «destinée », qui se singularise de la sorte. Le Monde du 10 juin 2001 rend compte de la présentation, à la télévision, d'une avocate des génocidaires rwandais lors de leur procès, dans les termes suivants :

La scène est un des grands moments du film [rhétorique du journaliste]. La caméra est derrière les visiteurs, à distance. Quand le gardien ouvre la première porte, Thierry de Lestrade ne filme pas ce qu'ils voient. Sa caméra est fixée sur les avocats seuls, de dos [rhétorique de la caméra], livrés à la tempête de leurs émotions [représentation de l' «émotion-source»]. Ne découvrir qu'après la cause de cette tempête - les cadavres amoncelés sur les tréteaux, les piles de crânes (têtes coupées à la machette), les squelettes de bébés - donne toute sa force à ce hors-champ [représentation de l'«émotion-source»]. Ce sont des hommes qui ont fait ça, répétera un des avocats, sous le choc. Les jours suivants, ils vont devoir aider des hommes soupçonnés des mêmes actes [rhétorique du journaliste].

On mesure ici combien le dispositif rhétorique mis en œuvre par le journaliste pousse le discours politique jusqu'aux limites de l'argumentation et de la communication.

La singularisation émotionnelle du discours politique, en même temps, est censée le rendre universel. Dans le même article sur le procès des supposés auteurs du génocide rwandais, il est fait état de la logique de l'avocate:

12. Le Monde, $1^{\mathrm{er}}-2$ octobre 2000 . Cet éditorial porte le titre suivant, particulièrement évocateur en matière d'émotion: «Ô Jérusalem».

13. Discours prononcé le 3 novembre 2000 à Groningen (Stichting Van der Leeuw-Lezing), et recueilli par Le Monde. 
Pour Laurence Dupuy Jauvert, il ne s'agit pas de défendre des salauds (la question lui parait révoltante), mais un principe: le droit pour chacun, quel qu'il soit et quoi qu'il ait commis, d'avoir un procès juste. (Le Monde, 10 juin 2001)

Le dispositif émotionnel nous renvoie à ces faits universels parce que fondateurs des logiques et des acteurs politiques : les droits de l'homme et la nécessaire institution des acteurs, des structures et des organes de leur défense. « $\mathrm{Ce}$ film remarquable, qui pose des questions de fond sur la notion de justice, poursuit Catherine Humblot dans cet article, interroge aussi la nature humaine et ses ombres. » Le sens de l'émotion dans les médias et le discours politique, se fonde, finalement, sur le fait que, si les appartenances ne sauraient être que culturelles et, par conséquent, localisées, c'est l'universalité qui caractérise la dimension singulière de l'identité et de la citoyenneté.

\section{L'émotion et la dialectique de l'identité}

La première signification de l'émotion se pense dans la problématique de l'identité, par ailleurs constitutive de la représentation médiatée du concept de sujet. Elle consiste dans la suspension de la dimension symbolique de l'identité singulière (perte de la singularité du sujet, par exemple dans les situations de foule ou de mouvements collectifs : manifestations de Berlin, lors de la destruction du Mur, ou de Belgrade, lors de la chute de Slobodan Milosevic). Elle peut consister aussi dans la suspension de la dimension de l'identité collectivetenance ou de sa solidarité, comme lors d'un deuil, ou d'une tragédie. Dans cette situation, l'identification au sujet ne repose pas sur une mise en scène distanciée de la subjectivité mais, au contraire, sur le fait qu'elle éveille la part non-symbolique de notre personnalité, celle qui ne fait pas de nous des sujets mais des personnes en proie au réel de leurs sentiments, de leurs passions, de leurs angoisses.

Dans l'information, l'émotion rend illisible cette relation particulière entre singularité et appartenance collective. Le film La chambre du fils, de Nanni Moretti (2001) ou l'image de la mère douloureuse du petit Mohamad, l'enfant palestinien, n'expriment pas l'appartenance; ils montrent la singularité de la situation des personnages, refoulant, en quelque sorte, la dimension collective de l'identité. C'est en cela que l'émotion est fondamentalement antinomique avec toute logique institutionnelle et toute signification proprement politique de l'information. Elle suspend la distanciation symbolique du fait politique dans les formes et les pratiques de l'information et de la communication. C'est pourquoi, dans de tels contextes, l'identité ne se fonde plus comme médiation entre la vérité singulière du sujet et la sociabilité politique de sa dimension collec- 
tive; elle se fonde exclusivement sur l'une ou sur l'autre. Les deux dimensions de l'identité du sujet - singulière et collective - sont, en quelque sorte, «décrochées» l'une de l'autre.

Dans l'expression singulière de l'émotion, le sujet est représenté comme confronté tout entier à l'impossibilité de dire son désir, ses sentiments, ses angoisses et, par conséquent, il est confronté à l'impossibilité de les inscrire dans la communication. L'émotion singulière peut, ainsi, expliquer des conduites individuelles qu'on ne peut interpréter, qu'on peut même à peine penser: «Sa douleur explose en haine, qu'elle jette sans retenue à la face des deux hommes », raconte Le Monde ${ }^{14}$, lors du procès, à propos de la mère d'un enfant violé et assassiné en Moselle. Dans l'émotion collective, les acteurs sont tout entiers confrontés à l'impossibilité de dire leur opinion, leur engagement, leur détermination. L'émotion collective renvoie à des actes collectifs incontrôlables et, à la limite, sans signification. On peut trouver un exemple de cela dans l'usage du mot, très fort, exaspération, dans le titre «Les Palestiniens entre frustration et exaspération ${ }^{15}$.

La représentation de l'émotion par les médias est aussi une expérience de leurs propres limites ${ }^{16}$, puisqu'elle brise la dialectique entre la dimension singulière de notre identité et sa dimension collective. Les médias représentent, en quelque sorte, l'impossibilité même de leur fonction, de leur pratique, de leur existence même. C'est pourquoi il y a peu de références à l'émotion dans les médias responsables, qui assument pleinement leur fonction et leur statut de médiation. Montrer l'émotion est le lot de ceux qui, à coup de sensationnel, de séduction, de populisme, viennent, au contraire, saper les logiques constitutives de la presse à scandales. En le faisant, l'émotion, les médias et les représentations esthétiques rendent possible la mise en scène, dans l'espace public, d'une forme de représentation métonymique de l'impossible à dire.

Il y a là à rendre compte de toute une rhétorique des médias, mais aussi d'un infléchissement de leur fonction idéologique et de la représentation qu'ils instituent, dans l'espace public, des identités politiques. Il s'agit de montrer comment les acteurs et les formes de la communication peuvent nous pousser jusqu'aux limites de la sociabilité, un peu comme l'hystérie (hustera, en grec: «le dernier point», «la limite») vient nous pousser, singulièrement, jusqu'aux limites de la subjectivité, et un peu, aussi, comme la violence vient nous pousser, collectivement, jusqu'aux limites de l'appartenance et de la sociabilité. En proposant, dans l'espace public, une mise en scène esthétique et symbolique

14. Le Monde, 31 janvier 2001.

15. Le Monde, 28 octobre 2000.

16. P. Sollers, 1971, L'écriture et l'expérience des limites, Paris, Seuil (Points). 
des limites qui nous instituent comme sujets sociaux, les médias et les représentations esthétiques mises en œuvre par la photo, le cinéma ou la littérature proposent une refondation du contrat social originaire. En marquant les limites au-delà desquelles, sans plus appartenir à la société, on se trouverait renvoyé à la douleur et à l'impossibilité de vivre dans une irréductible singularité.

\section{Résumé / Abstract / Compendio}

\section{Esthétique de la limite et dialectique de l'émotion}

La représentation de l'émotion dans les médias s'inscrit dans une esthétique du sujet dans sa singularité. En représentant l'émotion, les médias et les représentations esthétiques mises en œuvre par la photo, le cinéma ou la littérature proposent une refondation du contrat social originaire, en marquant explicitement les limites qui permettent de repenser la sociabilité.

Mots-clés : émotion, esthétique, identité, médias, analyse de discours, sociabilité.

\section{Esthetics of limits and dialectics of emotions}

The various representations of emotion in the mass media belong to the cesthetics of subjectivity. By representing emotions, mass media, photographs, cinema, literature make us restore the original "social contract», by clearly defining the limits which enable us to consider sociability in a new way.

Keywords : emotion, cesthetics, identity, media, discourse analysis, sociality.

\section{Estética del límite y dialéctica de la emoción}

La representación de la emoción en los mass media se integra en una estética de la subjetividad. Representando la emoción, los mass media, la fotografía, el cine o la literatura proponen una refundación del contrato social originario, marcando explicitamente los límites que permiten pensar de nuevo la socibilidad.

Palabras claves: emoción, estética, identidad, mass media, análisis de discurso, socibilidad. 